\title{
Uma ferramenta de análise e processamento de dados relacionados à avaliação da qualidade do ensino em $A V E$ 's
}

\author{
Artur O.R. Franco'1, Thiago Iachiley Araújo de Souza², Thomaz Edson Veloso da \\ Silva $^{3}$, Francisco Herbert Lima Vasconcelos ${ }^{3}$ \\ ${ }^{1}$ Instituto de UFC Virtual - Universidade Federal do Ceará (UFC) \\ Fortaleza - CE - Brasil \\ ${ }^{2}$ Departamento de Física - Universidade Federal do Ceará \\ Fortaleza - CE - Brasil
}

${ }^{3}$ Departamento de Engenharia de Teleinformática - Universidade Federal do Ceará

Fortaleza - CE - Brasil

artur.fhtagn@gmail.com, thomazveloso@virtual.ufc.br,

herbertevirtual.ufc.br, thiago.gil@hotmail.com

\begin{abstract}
In this paper, we present the implementation of a computational tool that provides a report of teaching indicators based on the evaluating of teaching effectiveness methodology. This tool was developed in Matlab ${ }^{\circledR}$ platform in order to provide to its handler faster data processing and the possibility of working with n-dimensional data structures. The development of a reporting tool that provides indicators of education is fundamental to aid the decision-making by the courses' managers. The results indicate the feasibility of using the tool in data related to online courses.
\end{abstract}

Resumo. Neste artigo, apresenta-se a implementação de uma ferramenta computacional que fornece um relatório de indicadores de ensino com base na metodologia de avaliação da efetividade do ensino. Esta ferramenta foi desenvolvida na plataforma Matlab ${ }^{\circledR}$ com o intuito de fornecer, ao seu manipulador, um rápido processamento dos dados e a possibilidade de trabalhar com estruturas de dados n-dimensionais. $O$ desenvolvimento de uma ferramenta que forneça relatórios de indicadores de ensino é fundamental para o auxílio à tomada de decisão por parte dos gestores dos cursos. Os resultados apontam para a viabilidade da utilização desta ferramenta em dados relacionados a cursos semipresenciais.

\section{Introdução}

A avaliação, de uma forma geral, não deve se restringir apenas ao fato de mensurar ou atribuir valores para determinados contextos. Esse processo implica em uma reflexão crítica sobre a prática que está sendo avaliada, auxiliando os gestores do processo na sua tomada de decisão [Silva et al, 2012].

Dentre os vários fatores que influenciam o processo avaliativo, destaca-se a necessidade de delimitar bem o que será avaliado. Pois, isto é um processo de decisão 
seletiva e que envolve relações subjetivas que muitas vezes as análises quantitativas não conseguem representar nessa subjetividade [Bassani e Behar, 2009].

Com a avalanche de dados obtidos e extraídos de Ambientes Virtuais de Ensino (AVE), diversos trabalhos [David et al, 2007][Vasconcelos et al, 2011][Almeida e Pimentel, 2010][Silva et al, 2012][Franco et al, 2013] procuraram analisar tais dados de modo a tentar compreender padrões existentes no comportamento dos alunos de cursos semipresenciais, e até mesmo auxiliar os gestores dos cursos a estabelecer melhores práticas ou metodologias de ensino.

No âmbito institucional, algumas Instituições de Ensino Superior (IES) apresentam aos alunos, tanto dos cursos presenciais quanto dos semipresenciais, um formulário de avaliação dos docentes. Esses formulários [Rêgo et al, 2011], eletrônicos ou impressos, fornecidos aos alunos procuram avaliar fatores relacionados à efetividade do ensino. A forma como a coleta de informações é feita e a quantidade de formulários coletados, tende a tornar-se um problema para o processamento dos dados obtidos [Vilela et al, 2012].

Neste sentido, o objetivo deste trabalho é o desenvolvimento de uma ferramenta computacional automatizada. Uma vez que esta dê suporte à análise de uma grande quantidade de dados, fornecendo um relatório com indicadores de ensino relacionados à metodologia de avaliação da efetividade do ensino (do inglês Students's Evaluation of Teaching Effectiveness - SETE) [Marsh e Bayle, 1993]. Neste artigo as imagens incluem análises práticas de algumas bases de dados tiradas de cursos semipresenciais e de um curso presencial a fim de garantir certa qualidade desta ferramenta.

\section{Trabalhos relacionados}

\subsection{Avaliação da Efetividade do Ensino}

Desenvolvido por Herbert Marsh [Marsh e Bailey, 1993], a metodologia SETE consiste em avaliar aspectos relacionados à efetividade do ensino. O SETE fornece indicadores de qualidade de ensino que permeiam aspectos subjetivos da aprendizagem [Marsh e Bailey, 1993].

Para a obtenção de dados relacionados à qualidade do ensino, foi desenvolvido o instrumento Student's Evaluating of Education Quality (SEEQ). Este tem sido utilizado em várias IES do mundo como principal instrumento para coleta de dados relacionado à opinião dos alunos sobre a efetividade do ensino [Roberts, 2007].

O SEEQ é constituído de 40 assertivas distribuídas em 11 fatores da aprendizagem, os fatores são: Aprendizagem, Entusiasmo, Organização, Interação de grupo, Relações individuais, Abrangência do conteúdo, Provas, Atribuições dos alunos, Análise global da disciplina, Análise global do professor e Características da disciplina e dos estudantes.

Para este trabalho foram usadas bases de dados com apenas as 31 primeiras assertivas do SEEQ, ou 29 nos casos em que não existem relações dos cursos/disciplinas com outras no sistema. As outras questões do instrumento de avaliação estão relacionadas à caracterização da amostra de forma subjetiva, não sendo possível 
sistematizar rótulos para essas informações pessoais [Franco et al, 2013]. Apesar de que a ferramenta oferece suporte para mais questões caso estas sejam obtidas.

\subsection{Análise estatística e computacional dos dados}

Diversos pesquisadores tem procurado encontrar métodos adequados de análise do SETE e do SEEQ e/ou têm tido feito analises usando tais instrumentos com as mais diversas técnicas. As técnicas utilizadas pelos pesquisadores vão desde análises de dados multivariados [Vasconcelos et al, 2013] até o uso de estatística descritiva [Franco et al, 2013].

Uma análise completa e simples do conjunto de dados pode ser obtida utilizando a abordagem da estatística descritiva pois essa, além de permitir, é mais acessível a um público maior e possui eficácia comprovada. Portanto foram usadas médias, variâncias, histogramas, boxplot e matrizes de correlação sendo que estas funções tiveram que ser refeitas e/ou adaptadas para nossa necessidade no Matla $^{\circledR}$. Isso se deve ao fato de que deve-se superar problemas como a incompletude dos dados comum neste tipo de base de dados [Franco et al, 2013].

\subsection{Requisitos de software}

No desenvolvimento de um software, torna-se importante realizar uma lista de requisitos concisos e ordenados a respeito do que um software deve possuir, para que ao final de sua implementação o cumprimento destes requisitos possam garantir todas as funcionalidades mínimas de forma correta, validando a ferramenta.

Os requisitos podem ser funcionais, onde definem comportamentos específicos e determinadas entradas [Sommerville et al, 2013], ou os requisitos podem ser não funcionais, que determinam restrições ao processo de desenvolvimento e aos seus padrões, eles não retratam pontos específicos das funcionalidades de um sistema [Sommerville et al, 2013].

\section{Metodologia}

\subsection{Matlab ${ }^{\circledR}$}

O programa foi desenvolvido na plataforma $M a t l a b^{\circledR}$, a qual é conhecida no meio acadêmico pela sua praticidade ao lidar com dados e por possuir uma linguagem de programação própria e de fácil uso. Esta plataforma inclui recursos para que se possa trabalhar com interface gráfica chamadas de (GUIDE) Graphical User Interface Design Environment e as interfaces por ele criadas são chamadas de (GUI) Graphical User Interface. Isso possibilita a manipulação de dados sem a necessidade de programar diretamente no prompt de comando. Logo, o código-fonte do programa apresentado neste trabalho é interpretado diretamente pelo Matlab.

\subsection{Requisitos e definições da ferramenta}

A lista de requisitos é embasada pelo trabalho do Franco (2013) onde sua análise não necessita de um profundo conhecimento em estatística, seu maior problema foi a organização e tratamento dos dados. Devido a isso, o primeiro requisito é o tratamento 
sem o conhecimento prévio de programação ou de sua lógica e foram embasados nestas ideias. Destacam-se como requisitos funcionais (RF):

1. A base de dados deve ser gerada a partir de um arquivo do tipo planilha eletrônica $(. x l s, . x l s x$ ou .ods);

2. Deve-se ter um recurso para gerar um relatório completo em utilizando a base de dados carregada.

A seguir, os requisitos não funcionais (RNF):

1. O recurso deve ser realizado em $M a t l a b^{\circledR}$ com interface gráfica para uso de leigos em programação;

2. Deve ser capaz de realizar operações de médias e variância mesmo com a incompletude da base de dados;

3. O usuário deve ser capaz de definir o nome da base de dados em separado da turma;

4. Deve-se poder inserir quantos cursos quiser por turma, bem como quantos alunos desejar.

Tendo o RF-1 em vista faz-se necessário à determinação da organização dos dados obtidos na aplicação do SETE nos arquivos da planilha eletrônica. Eles devem respeitar a seguinte estrutura: cada planilha de um arquivo será um aluno, onde cada linha representa uma questão e cada coluna representa uma disciplina. O uso da ferramenta é generalizado, observa-se que ela permite o uso de quantas disciplinas ou questões forem necessárias. Este padrão deve ser adotado por qualquer ferramenta do gênero para que as mesmas tornem-se realmente funcionais nos mais diversos âmbitos e aplicações. Evitando desta forma trabalhos excessivos de correções e atualizações futuras para casos específicos.

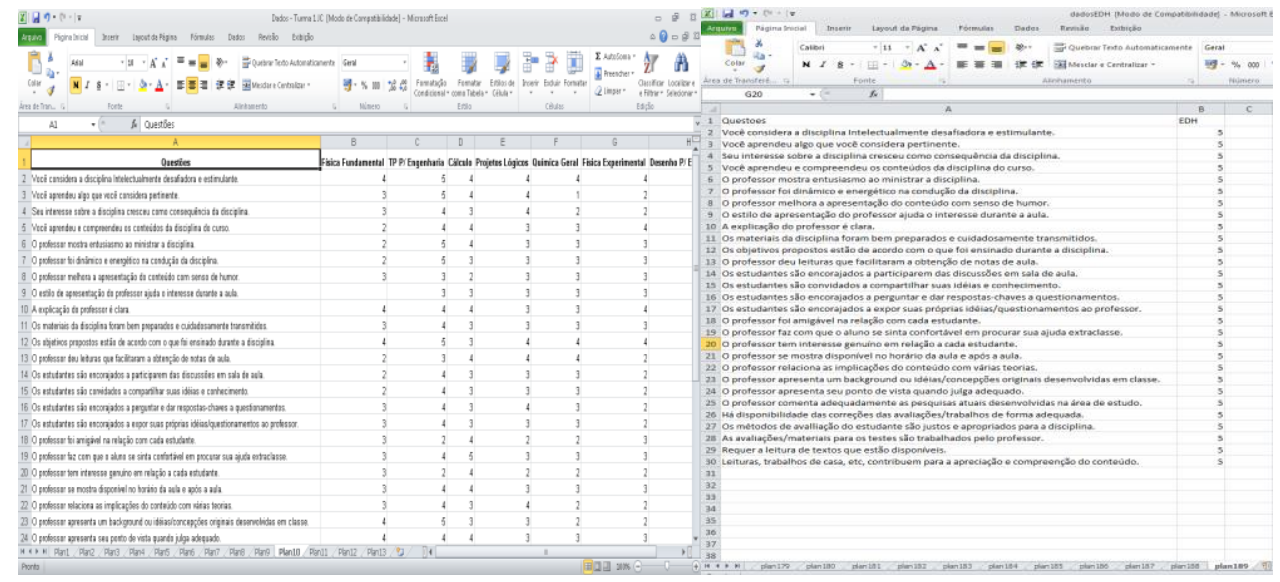

Figura 1. Exemplo de base de dados

\section{Resultados}

\subsection{Geral sobre a ferramenta - Funcionamento}

A ferramenta gerada deve ser capaz de carregar e de tratar a base de dados, retornando algumas características diretas e gerais. Tais como, número de alunos, de disciplinas e o 
percentual de questões respondidas (figura 2). Além destas informações iniciais deve-se ser capaz de gerar um relatório externo com as ferramentas estatísticas citadas na subseção 3.2 deste trabalho.

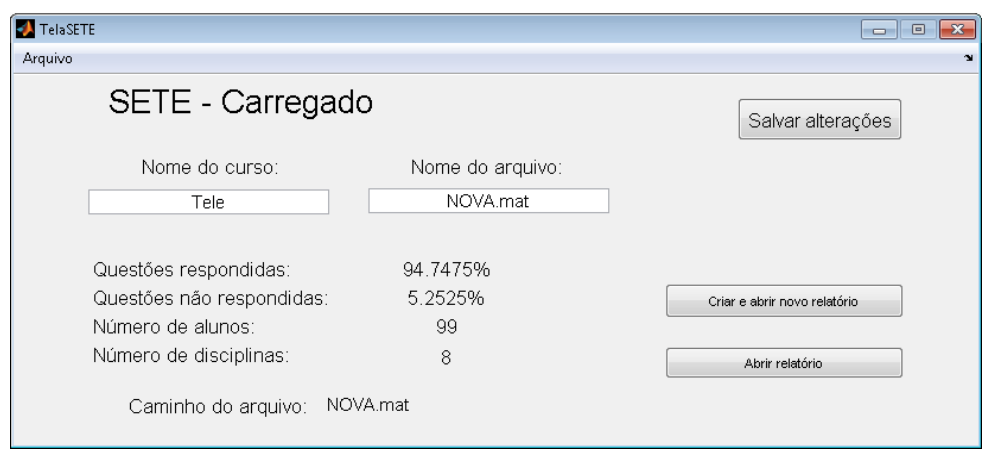

Figura 2. A tela da ferramenta após carregar uma base

Observa-se que a ferramenta possui a capacidade de gerar uma nova base de dados a partir do arquivo em algum formato das planilhas citadas gerando um arquivo de formato Matlab Data (.mat). O Matlab Data será a nova base de dados e possuirão os dados originais reorganizados mais os termos das disciplinas, o nome da base de dados e alguns resultados de outros tratamentos realizados na base (ver na Figura 2). Deve-se notar que além de gerar uma nova base de dados ele pode adicionar mais dados a estes, de outros arquivos do formato da Planilha de pasta do Excel, ou equivalente, clicando em "Arquivo > Adicionar base de dados", digitando Ctrl $+D$ (veja na Figura 3). Caso gerente já tenha gerado uma base de dados e queira reabri-la. Após realizar tais operações o usuário pode ou não querer salvar suas ações. Isto é importante porque dá certa integridade em relação aos dados e permite fazer várias análises ao longo do tempo.

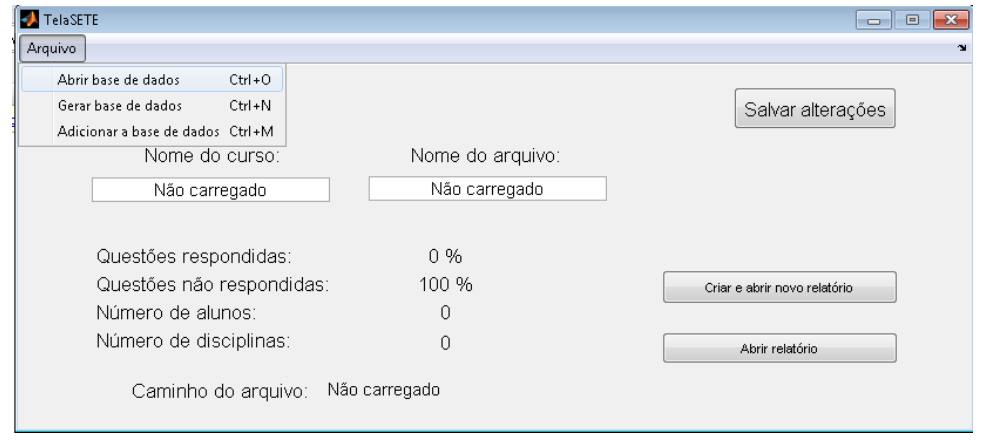

Figura 3. A tela da ferramenta após carregar uma base

\subsection{Relatório da ferramenta}

O programa permite ao usuário gerar um relatório escrito no formato Hiper Text Markup Language (HTML), ou seja, ele poderá ser aberto em qualquer navegador que o computador por ventura venha a ter. Isto é importante porque o HTML não necessita ter uma ferramenta incomum no computador como um leitor de arquivos Portable Document Format (PDF), mesmo que hoje a grande maioria das máquinas tenha leitores de PDF. Além do mais o próprio Matlab ${ }^{\circledR}$ possui um navegador que pode interpretar o arquivo HTML. 
O relatório possui uma página inicial com os dados gerais da base de dados, bem como as médias e variância da pontuação de cada disciplina. Abaixo há a matriz de correlação dos fatores (Figura 4). Abaixo dele existem alguns gráficos do tipo boxplot e histograma que dão uma vista geral da distribuição dos dados. Contudo, o boxplot desta página permite ver os resultados das disciplinas de forma comparativa, tanto nas questões do SETE quanto nos fatores do SEEQ.

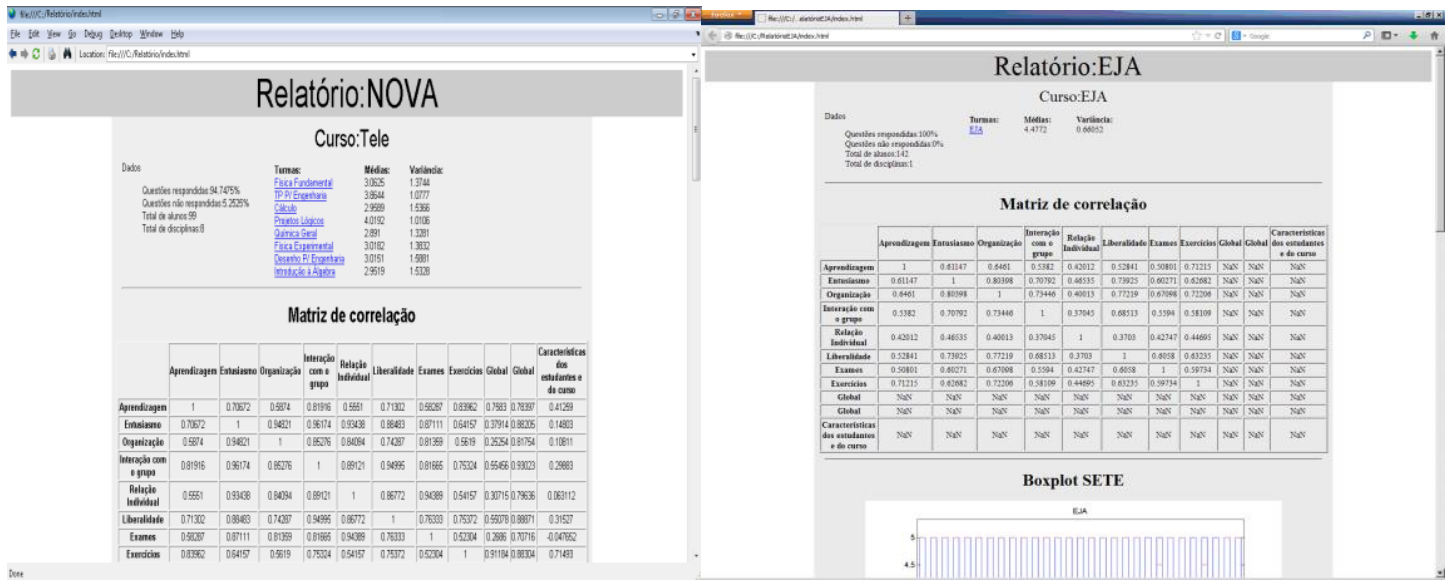

Figura 4. Parte da tela inicial do relatório

Além da página inicial (veja a Figura 4) existe uma página para cada disciplina semelhante a esta apresentada. Contudo, cada disciplina abordará uma análise mais detalhada referente aos fatores. Assim seus gráficos apontam relações e comparações dos fatores referentes apenas para aquela disciplina e não do todo (veja o exemplo na Figura 5).
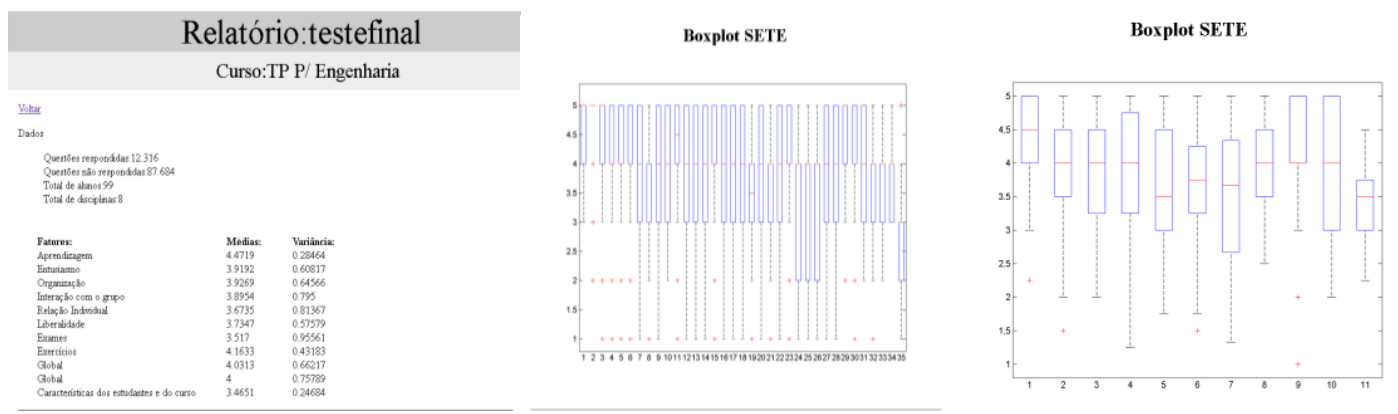

Figura 5. A tela do relatório de uma turma

\subsection{Revisão do projeto e Validação}

É importante que um software tenha seu funcionamento analisado a fim de encontrarem falhas e pontos de melhorias que tornem o seu uso mais fácil, seguro e rápido. No caso dos AVE especificamente nota-se a capacidade de reuso e a adaptabilidade são de grande importância devido a grande pluralidade de sistemas do gênero. Logo, deve-se adotar um processo para o desenvolvimento e validação da melhor forma possível do software desenvolvido. Mesmo que este, não alcance um grau de perfeição algo que é praticamente impossível para qualquer software [Vasconcelos et al, 2006]. Observa-se que o recurso cumpre com esmero todos os requisitos estipulados. Notavelmente 
procura-se obter certo grau de qualidade neste trabalho mesmo havendo a característica de protótipo.

Contudo, o uso do termo qualidade não deve ser usado de forma trivial e nem geral [Vasconcelos et al, 2006]. Consideram-se como qualidades a clareza, a concisão, estilo, precisão, completeza do código como inerentes ao projeto [Sommerville et al, 2007]. Pode-se observar isso, porque devido à facilidade de implementação no Matlab $^{\circledR}$, este garante toda a autonomia necessária para uso da ferramenta e a reutilização de código. Incluindo no que condiz as funções das análises, cálculos e para escrever o próprio relatório. A escrita do relatório é feita carregando trechos modulares de informação a respeito dos cursos e o texto que aparece é carregado do arquivo com os dados obtidos da análise (o funcionamento deste está ilustrado na figura 6). Isto condiz com uma alta modularização do recurso. Isto é bastante notável, porque as características e módulos ajustáveis que serão escritos no relatório ficam armazenadas no arquivo site.mat que independe da base de dados adotada. Mas, que também não dificulta seu acesso ou troca por trechos de configurações do HTML, permitindo uma fácil manutenção. $\mathrm{O}$ mesmo pode-se dizer das funções armazenadas nos diretórios (a)double, que possuem como entrada valores numéricos como a base de dados, e o diretório@string que possui funções referentes ao tratamento de texto usado para escrever os resultados no relatório.

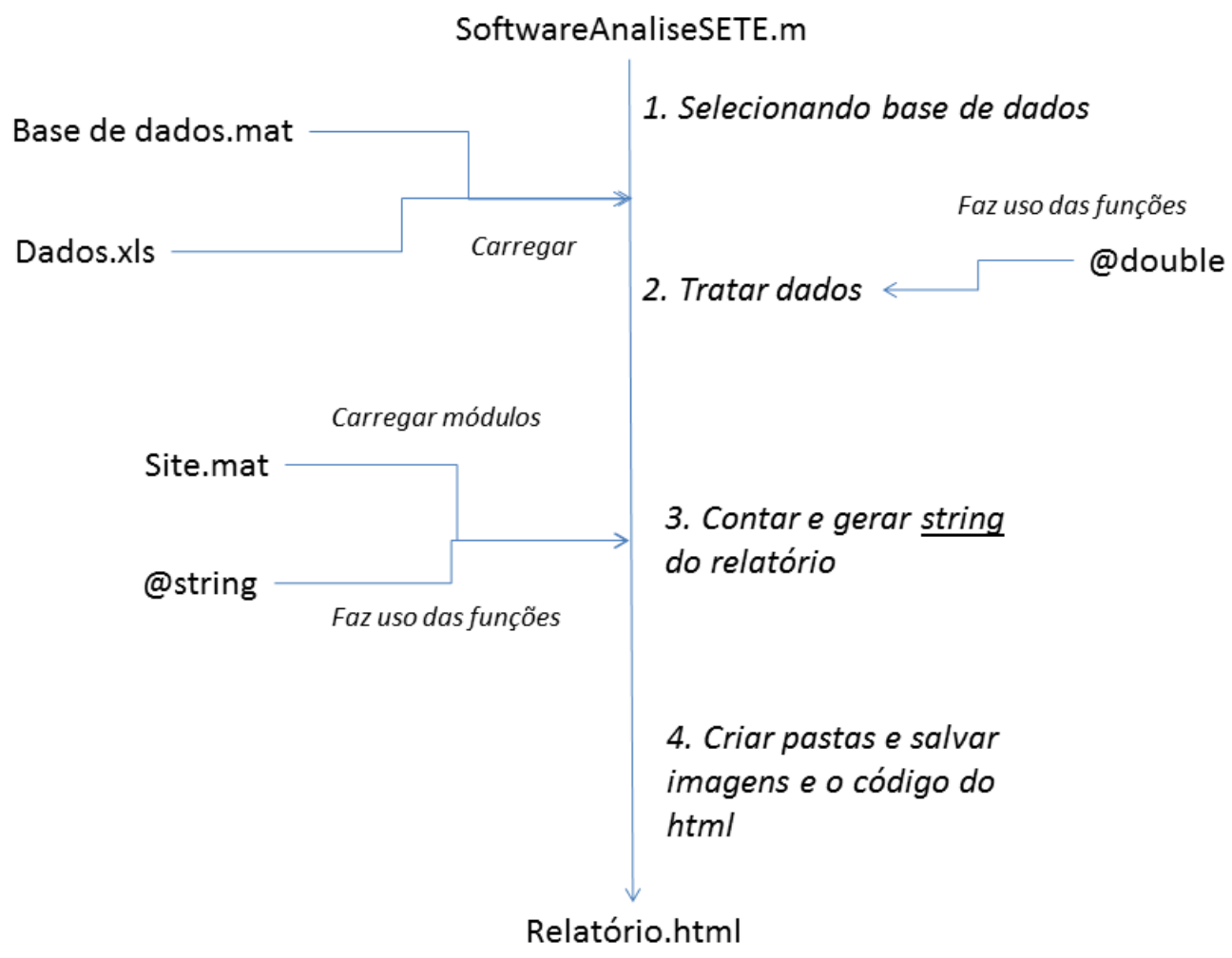

Figura 6. Processamento do GUIDE 
Este software agrega alguns tipos de arquivos diferentes, contudo o uso do $M_{a t l a b}{ }^{\circledR}$ como tecnologia de suporte e desenvolvimento garante uma leitura e processamento dos dados de forma bastante flexível. Em resumo, o software no Matlab $^{\circledR}$ será capaz de garantir o carregamento ou adição dos dados das planilhas de diversos formatos em uma base de dados que apenas o Matlab ${ }^{\circledR}$ é capaz de acessar, que pode ser compartilhada de forma fácil por ser um arquivo pequeno. Isto garante uma boa integridade dos dados. Em uso destes dados carregados o programa gerará um relatório que pode ser visto pelo analista, e compartilhado ou armazenado (veja a Figura 7). Salienta-se que mesmo o relatório sendo do tipo HTML, ele pode ser aberto no Matlab $^{\circledR}$ por um navegador (ou browser) próprio dele ou qualquer outro navegador do usuário, inclusive sendo compatível para compartilhamento web pelo próprio sistema (caso o Matlab $^{\circledR}$ esteja integrado ao sistema).

Por fim, o uso da ferramenta foi aplicado em três cursos semipresenciais distintos, mais especificamente nos cursos de Educação Ambiental (EA), Direitos Humanos (DH) e Educação para Jovens e Adultos (EJA). Todos os dados foram usados independentemente por serem cursos descorrelacionados. A fim de testar a adaptabilidade do programa neste foi aplicado uma base de dados com seis disciplinas do curso de Engenharia de Teleinformática. Estas aplicações são adotadas como imagens de exemplo neste trabalho. Todas as estas obtiveram bom desempenho do programa, infelizmente não faz parte do escopo do artigo estudar casos da aplicação, para maiores detalhes deste gênero recomendam-se [Franco et al, 2013] uma vez que esta publicação possui especificações da análise adotada.

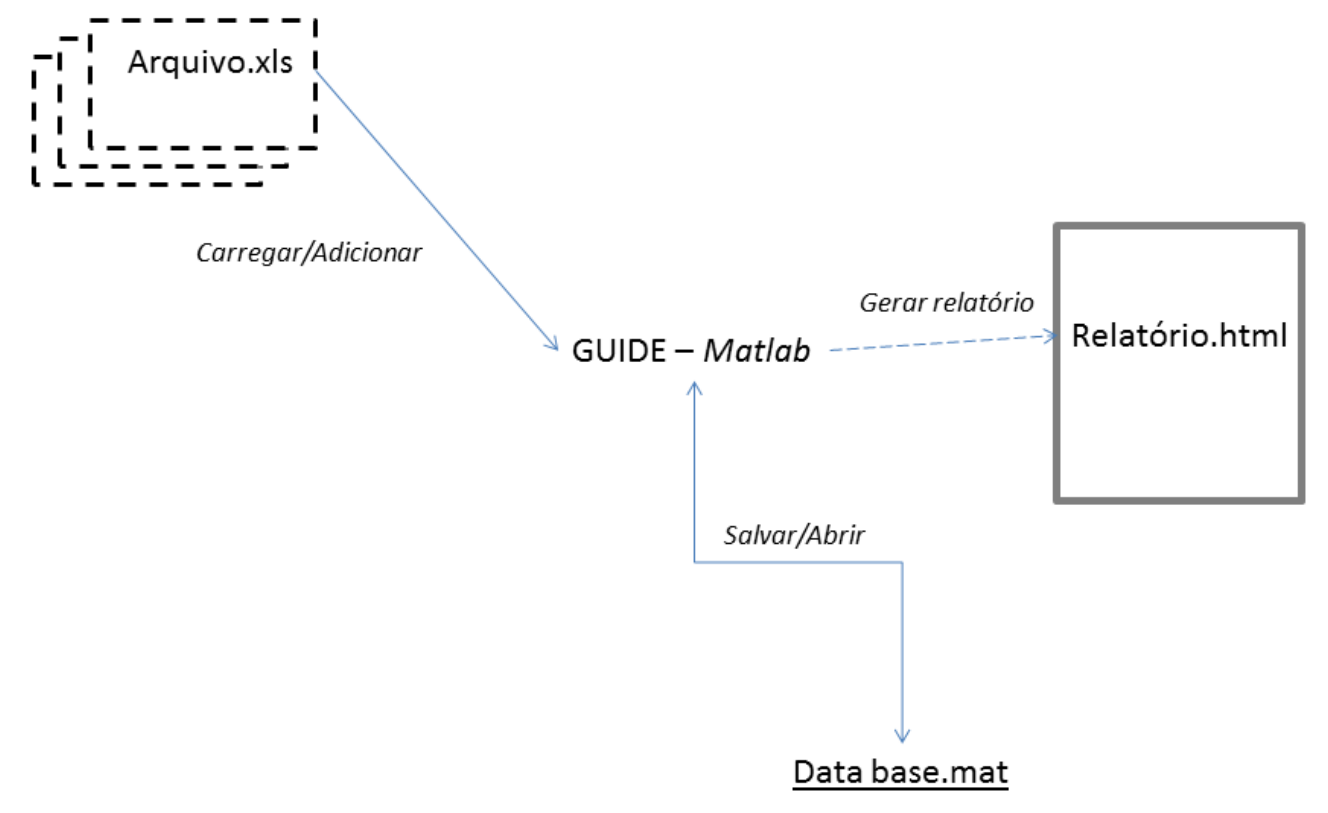

Figura 7. Funcionamento do software de análise 


\section{Conclusão}

Conclui-se deste trabalho que uma padronização no tratamento e análise de dados subjetivos dos professores é uma atividade complexa. Assim como, a análise de avaliação de atividades do aluno [Dario et al, 2009]. Contudo, passível de ser realizada.

O software abordado procurava manter uma simplicidade em seu uso em prol do foco de especificar o tratamento e a sua implementação. Portanto, o que foi apresentado é uma boa orientação para aqueles que pretendem fazer uso do controle da qualidade de ensino independente do seu AVE. Atualmente os recursos de JavaScript tem tido melhorias significativas sendo plausível o desenvolvimento de uma ferramenta integrada aos sistemas web, de forma genérica, para análise que seria um dos próximos passos deste trabalho.

Tendo em vista a análise da qualidade de ensino do professor, deve ser interessante também avaliar a qualidade das ferramentas e suas relações diretas com os desempenhos e comportamentos dos alunos. Neste caso pode-se utilizar de uma nova estrutura de validação de software para avaliação. Bem como fornecer vários novos dados que relacionem o desempenho e comportamento do instrutor com o desempenho e melhoria do aluno, permitindo a construção de sistemas inteligentes que auxiliem no processo de ensino e no controle de qualidade do mesmo.

\section{Referências}

Franco, A. O. R., Silva, T. E.V., Vasconcelos, F. H. L., Paiva, I. R. (2013), "Evaluating the Students' Perception of the Teaching Effectiveness in a Teleinformatics Engineering”. In: IEEE Educon Conference - Berlim, 2013.

Marsh, H. W., M. Bayle, (1993), "Multidimensional Students' Evaluations of Teaching Effectiveness: A Profile Analysis", The Journal of Higher Education, Vol. 64, No. 1.

Roberts, T., (2007), "Teaching Learning: SEEKing useful feedback from students" Paideia, Vol. 2, No. 1.

Vasconcelos, F. H. L., Silva, T. E. V., Gomes, P. R. B., Nunes, A. O., Andriola, W. B., Almeida, A. L. F., Mota, J. C. M. (2011) “Análise do Desempenho Discente em um Ambiente Virtual de Aprendizagem Através de Decomposições Tensoriais Multilineares". In: Anais do XXII SBIE - XVII WIE.

Vasconcelos, A. M. L., et al. (2006), "Introdução à engenharia de software e à qualidade de software". Tese de Pós-Graduação - UFLA - Lavras, MG.

Rocha, A. R., Campos, G. H. B. (1993), "Avaliação da qualidade de software educacional". Revista "Em Aberto".

Vilela, R. F., Valle, P. H. D., Muniz, R. J., Lima, W. A., Inocêncio, A. C. G., Junior, P. A. P. (2012), "SCATeDi: Sistema Inteligente para Avaliação de Desempenho Escolar em Avaliações Discursivas." Anais do XVIII WIE.

Sommerville I. F., et al. (2007), "Engenharia de Software". Editora Pearson. Addison Wesley, $8^{\circ}$ Edição.

Dario D., Rocha, H. V., Otsuka J., Silva, A. C., Cavalcante, C., Gavilán, A. M. C., (2009) "Aplicação de Técnicas de Visualização de Informação em Ferramentas para 
Apoio à Avaliação Formativa em Sistemas de Ensino a Distância", Simpósio Brasileiro de Informática na Educação, WAvalia II, Florianópolis - SC.

Rêgo, A. H. G., Gomes, A. V., Ana, M. L., Brito, A. C., (2011) "Um módulo gerador de relatórios no auxílio às atividades docentes no Moodle". Anais do XXII SBIE - XVII WIE, IV WAvalia, 2011.

David, P. B., Pequeno, M. C., da. Silva, A., Souza, C. F., Júnior, G. S. V., de Castro Filho, J. A., Ventura, P. P. B., e Maia, S. (2007). Avaliação da aprendizagem em educação a distância numa perspectiva sócio-interacionista. Anais do XVIII SBIE.

Bassani, P. S. e Behar, P. A. (2009). Modelos Pedagógicos em Educação à Distância. Avaliação da Aprendizagem em Ambientais Virtuais. Artmed.

Almeida, A. e Pimentel, E. P. (2010). "Mineração de dados no ensino a distância para fins de avaliação do uso das ferramentas de aprendizagem do ambiente tidia-ae". In: Anais do III WAvalia.

Silva, T. E. V., Souza, T. I. A., Barros Filho, F. F., Santos, F. J., Gomes, P. R. B., Ribeiro, G., Nunes, A. O., Vasconcelos, F. H. L. (2012) "Análise de Componentes Principais Aplicada a Avaliação Discente: Um Estudo de Caso em Ambientes Virtuais de Aprendizagem”. In: Anais do Computer on the beach.

Vasconcelos, F.H.L., Silva, T. E. V., Almeida, A. L. F., Mota, J. C. M., Andriola, W. B. (2013). "Multilinear Decomposition Application into Students' Evaluation of Teaching Effectiveness". In: IEEE Educon Conference - Berlim, 2013. 